\title{
Principal component analysis and comprehensive evaluation on drought tolerance difference of canola cultivars at germination and emergence stages
}

\author{
Rui Wang ${ }^{1 *}$, Wenli Peng ${ }^{1}$, and Weixian $\mathrm{Wu}^{1}$ \\ ${ }^{1}$ Tongren University, Tongren, Guizhou 554300, China. *Corresponding author (ngywr@gztrc.edu.cn). \\ Received: 6 April 2021; Accepted: 15 July 2021; doi:10.4067/S0718-58392021000400557
}

\begin{abstract}
Drought stress in autumn is an important factor restricting canola (Brassica napus L.) germination and emergence in the Yangtze River basin in China. Screening drought tolerance varieties in canola is a cost-effective and practical way. The objective of this study was to explore the methods of drought tolerance evaluation and to screen canola varieties with drought tolerance at germination and emergence stages. The experiments were conducted with 53 cultivars under drought treatment and non-stress control. In this research, 16 investigated indicators were synthesized into six independent indices which could represent $87.5 \%$ characters information related to drought resistance of canola varieties. With this analysis and evaluation, 53 varieties could be classified into three categories: five varieties as YY10, JLYT, LY9, ZS11, and YY50 owned drought-resistant; 16 varieties as ZY589, QZ7 and DAZ600 and so on, were moderately drought-resistant; and the other 32 varieties belonged to non-drought-resistant. The study proved that there were six indices as mean germination time, germination index, seedling emergence time, fresh and dry weight per plant and 1000-grains weight, which could be used as identifying indicators related to crop drought resistance at germination and seedling stage.
\end{abstract}

Key words: Canola, drought resistance, germination, principal component analysis, seedling.

\section{INTRODUCTION}

Southwest China is an important winter canola (Brassica napus L.) planting area located in the upper reaches of the Yangtze River (Yin and Wang, 2012). Although the annual precipitation is high during winter canola growing season in this region, the precipitation periods are uneven which have led to seasonal drought frequently (Aksenova et al., 1994; Bai et al., 2014). Seasonal rainfall shortage is a main factor limiting the plant growing and seedling establishment for autumn sowing canola, and canola yield is considerably influenced in drought autumn which commonly coincided with the canola sowing time in this region (Li et al., 2015; Channaoui et al., 2019). In addition, during canola seedling stage, seasonal drought would lead to seedling emerging unevenly, slow emergence of leaves, reduction of green leaf area, which seriously affects harvesting yield and seed quality (Xie et al., 2013; Bai et al., 2014; Li et al., 2015; Jian et al., 2016). Seed germination and successful seedling establishment are two important prerequisites for yield determination (Xie et al., 2013; Bai et al., 2014). Hence, varieties with high germination potential and seed vigor should be identified to ensure optimum seedling establishment for high seed yield (Maataoui et al., 2005; Nourgholipour et al., 2018). Therefore, it is the top priority for screening of canola genotypes with drought tolerance at germination stage and evaluation of selecting indices for canola production.

In recent years, different methods have been reported for crop drought tolerance identification, which are involved in the physiological, biochemical, morphological identification indicators (Li et al., 2010; Xie et al., 2013; Bai et al., 2014; Li et al., 2015; Jian et al., 2016; Channaoui et al., 2019). On the one hand, canola variety with drought resistance 
could be identified by some single indicator, but be vulnerable to environmental difference due to the combinative and comprehensive characters determining canola drought resistance (Xie et al., 2013; Jian et al., 2016). On the other hand, only using the method of membership function to evaluate multiple indicators would lead to the overlapping of information provided by each single indicator; because there has been different correlation among different indicators, it would be difficult to obtaining a concise and convenient rule for canola drought resistance identification (Li et al., 2010; Bai et al., 2014; Channaoui et al., 2019). Principal component analysis could integrate a linear combination of multiple indicators into fewer comprehensive indices. There is no correlation among these comprehensive indicators, which could present overall information from the original multiple indices related to canola drought resistance. It is gradually applied to evaluate some crops drought tolerance comprehensively (Liu et al., 2016; 2019a; Pavia et al., 2019).

Some researchers have already focused on screening a single indicator to identify canola varieties with drought tolerance. In our research, 53 canola varieties with different drought resistance in the Yangtze River Basin were analyzed through the correlation analysis, principal component analysis, membership function and cluster analysis. Much drought resistance indices were weighted and analyzed to obtain the comprehensive evaluation value of drought resistance for the experimental varieties. The purpose of this study aimed to provide an efficient method selecting drought-resistant genotypes in winter canola. At the same time, this study could also provide theoretical reference for drought resistance breeding and canola production in the Yangtze River Basin of China.

\section{MATERIALS AND METHODS}

\section{Plant material}

The experiment was carried out at Tongren University in China. In this experiment, 53 canola (Brassica napus L.) varieties were chosen, which have been widely planted in the planting areas of the Yangtze River basin. Seeds of these 53 varieties were collected, dried and stored in a refrigerator at $4{ }^{\circ} \mathrm{C}$ until this experiment. The studied canola varieties were provided by the College of Plant Science and Technology in Huazhong Agricultural University and were numbered from 1 to 53, and these varieties were as follows (in the bracket, every variety was represented with seed yields, phenological periods, sowing dates in the field and suitable planting regions):

(1) ZYY8 (widely planted in the upper reaches of the Yangtze river of China, which seed yield was about $2983 \mathrm{~kg} \mathrm{ha}^{-1}$ with a growing period of $219 \mathrm{~d}$ after sowing (das) at any time from October 1 to 10), (2) YY57 (upper reaches of the Yangtze river, seed yield about $2916 \mathrm{~kg} \mathrm{ha}^{-1}$ with 218 das at any time from October 1 to 15), (3) YY817 (upper reaches of the Yangtze river, seed yield about $2670 \mathrm{~kg} \mathrm{ha}^{-1}$ with 222 das growing period at any time from October 15 to 30), (4) YY10 (upper reaches of the Yangtze River, about $2381 \mathrm{~kg} \mathrm{ha}^{-1}$ with 214 das from October 1 to 10), (5) ZY589 (in the middle and lower reaches of the Yangtze river, about $2295 \mathrm{~kg} \mathrm{ha}^{-1}$ with 217 das from October 1 to 10), (6) ZY28 (in the middle and upper reaches of the Yangtze river, about $2587 \mathrm{~kg} \mathrm{ha}^{-1}$ with 212 das from October 1 to 15), (7) FY520 (in the middle and upper reaches of the river, about $2559 \mathrm{~kg} \mathrm{ha}^{-1}$ with 218 das from October 1 to 10), (8) ZNY9 (in the middle and upper reaches, about $2742 \mathrm{~kg} \mathrm{ha}^{-1}$ with 232 das from October 5 to 15), (9) YG2009 (in the middle and upper reaches, about 2470 $\mathrm{kg} \mathrm{ha}^{-1}$ with 217 das from October 5 to 15), (10) YY50 (in the middle and upper reaches, about $2319 \mathrm{~kg} \mathrm{ha}^{-1}$ with 219 das from October 1 to 10), (11) ZYZ19 (in the lower reaches, about $2930 \mathrm{~kg} \mathrm{ha}^{-1}$ with 230 das from October 1 to 15), (12) ZS12 (in the middle and upper reaches, about $3135 \mathrm{~kg} \mathrm{ha}^{-1}$ with 230 das from October 1 to 10), (13) ZS9 (in the middle and lower reaches, about $2385 \mathrm{~kg} \mathrm{ha}^{-1}$ with 220 das from September 15 to 30), (14) ZYZ7 (in the middle and lower reaches, about $2520 \mathrm{~kg} \mathrm{ha}^{-1}$ with 219 das from September 20 to October 5), (15) ZS11 (in the middle and lower reaches, about $2479 \mathrm{~kg}$ ha $^{-1}$ with 234 das from September 25 to October 10), (16) CZY3 (in the middle reaches, about $2525 \mathrm{~kg} \mathrm{ha}^{-1}$ with 217 das from September 20 to October 5), (17) FY792F1 (in the middle reaches, about $2325 \mathrm{~kg} \mathrm{ha}^{-1}$ with 216 das from September 20 to October 20), (18) XZY814 (in the middle reaches, about $2608 \mathrm{~kg} \mathrm{ha}^{-1}$ with 220 das from September 20 to October 10), (19) HXY12 (in the middle and lower reaches, about $2761 \mathrm{~kg} \mathrm{ha}^{-1}$ with 219 das from October 5 to 15), (20) BYZ12 (in the upper reaches, about $2235 \mathrm{~kg} \mathrm{ha}^{-1}$ with 190 das from October 10 to 25), (21) JXY11 (in the middle reaches, about 2427 $\mathrm{kg} \mathrm{ha}^{-1}$ with 218 das from September 15 to October 5), (22) CZY2 (in the middle reaches, about $2422 \mathrm{~kg} \mathrm{ha}^{-1}$ with 215 das from September 20 to October 15), (23) CYZ83 (in the middle and lower reaches, about $2578 \mathrm{~kg} \mathrm{ha}^{-1}$ with 215 das from September 15 to October 15), (24) SXY9 (in the middle reaches, about $2362 \mathrm{~kg} \mathrm{ha}^{-1}$ with 220 das from September 25 to October 30), (25) LY9 (in the middle reaches, about $2618 \mathrm{~kg} \mathrm{ha}^{-1}$ with 218 das from October 15 to 25), (26) QY7 (in the 
Yangtze river basin, about $3000 \mathrm{~kg} \mathrm{ha}^{-1}$ with 225 das from September 25 to October 10), (27) RHY6 (in the lower reaches, about $3231 \mathrm{~kg} \mathrm{ha}^{-1}$ with 226 das from October 5 to 15), (28) KLY1 (in the lower reaches, about $2562 \mathrm{~kg} \mathrm{ha}^{-1}$ with 204 das from September 25 to October 10), (29) BY517 (in the upper reaches, about $2528 \mathrm{~kg} \mathrm{ha}^{-1}$ with 224 das from October 1 to 10), (30) GYZ6 (in the middle reaches, about $2025 \mathrm{~kg} \mathrm{ha}^{-1}$ with 202 das from October 5 to 15), (31) CJZY3 (in the upper reaches, about $2792 \mathrm{~kg} \mathrm{ha}^{-1}$ with 214 das from September 25 to October 10), (32) QDJ (in the middle and upper reaches, about $2823 \mathrm{~kg} \mathrm{ha}^{-1}$ with 208 das from October 1 to 10), (33) CSY (in the middle and upper reaches, about $2856 \mathrm{~kg} \mathrm{ha}^{-1}$ with 208 days after sowing from October 1 to 10), (34) JLY5 (in the upper reaches, about $2715 \mathrm{~kg} \mathrm{ha}^{-1}$ with 210 das from September 25 to October 10), (35) JLY7 (in the upper reaches, about $2737 \mathrm{~kg} \mathrm{ha}^{-1}$ with 216 das from September 25 to October 10), (36) JLY10 (in the upper reaches, about $2802 \mathrm{~kg} \mathrm{ha}^{-1}$ with 210 das from October 1 to 10), (37) YBS (in the upper reaches, about $2887 \mathrm{~kg} \mathrm{ha}^{-1}$ with 210 das from October 5 to 15), (38) JLYT (in the upper reaches, about $2730 \mathrm{~kg} \mathrm{ha}^{-1}$ with 210 das from October 5 to 15), (39) YB (in the upper reaches, about $2718 \mathrm{~kg} \mathrm{ha}^{-1}$ with 210 das from October 5 to 15), (40) DAZ600 (in the Yangtze river basin, about $2875 \mathrm{~kg} \mathrm{ha}^{-1}$ with 210 das from October 1 to 10), (41) XDZY9 (in the upper reaches, about $2794 \mathrm{~kg} \mathrm{ha}^{-1}$ with 227 das from October 10 to 25), (42) DZY10 (in the Yangtze river basin, about $2455 \mathrm{~kg}$ ha $^{-1}$ with 224 das from October 1 to 10), (43) JHH (in the upper reaches, about $2250 \mathrm{~kg} \mathrm{ha}^{-1}$ with 190 das from October 20 to 30), (44) DZY5 (in the middle and upper reaches, about $2473 \mathrm{~kg} \mathrm{ha}^{-1}$ with 223 das from September 10 to October 10), (45) XY50 (in the middle reaches, about $2340 \mathrm{~kg} \mathrm{ha}^{-1}$ with 216 das from September 20 to October 10), (46) ZYZ108 (in the lower reaches, about $3094 \mathrm{~kg} \mathrm{ha}^{-1}$ with 226 das from October 10 to 25), (47) ZY18 (in the lower reaches, about $2221 \mathrm{~kg}$ ha $^{-1}$ with 219 das from October 15 to 30), (48) YY9 (in the upper reaches, about $2298 \mathrm{~kg} \mathrm{ha}^{-1}$ with 220 das from October 1 to 10), (49) BDHZ (in the middle reaches, about $2100 \mathrm{~kg} \mathrm{ha}^{-1}$ with 188 das from October 20 to 30), (50) FYJ (in the upper reaches, about $2725 \mathrm{~kg} \mathrm{ha}^{-1}$ with 220 das from September 15 to October 15), (51) YB (in the Yangtze river basin, about $2745 \mathrm{~kg} \mathrm{ha}^{-1}$ with 225 das from October 5 to 15), (52) QY20 (in the upper reaches, about $2260 \mathrm{~kg} \mathrm{ha}^{-1}$ with 235 das from September 15 to October 10), (53) QZ7 (in the upper reaches, about $2485 \mathrm{~kg} \mathrm{ha}^{-1}$ with 195 das from April 5 to May 10).

\section{Experimental design and management}

The experiment was a two-factor in a completely randomized design with five replicates for each treatment. The mainfactor was the two water potential treatments, including no drought stress (control) which was the nutrient solution of Hoagland; and drought stress treatment simulated by adding 10\% polyethylene glycol (PEG6000, $10 \% \mathrm{in} \mathrm{w/v,} \mathrm{MW)} \mathrm{to} \mathrm{the}$ Hoagland nutrient solution (-0.15 MPa, Michel and Kaulmann, 1973). The concentration of 10\% PEG6000 was based on the results of our previous research on the same canola cultivars, where seeds germination was affected at water potentials lower than the solution concentration of $10 \%$ PEG6000. Sub-factor was different canola varieties.

Germination trials were carried out in polyethylene boxes $(12 \mathrm{~cm}$ length $\times 12 \mathrm{~cm}$ width $\times 6 \mathrm{~cm}$ height), which were sterilized with $1 \% \mathrm{NaClO}$ solution for $15 \mathrm{~min}$ and rinsed before use. Three sheets of sterile filter paper were moistened with $10 \mathrm{~mL}$ designed solution and placed in the bottom of every germination box. For each variety, the control treatments were Hoagland nutrient solution and the drought stress treatments were 10\% PEG in Hoagland solution. The experimental seeds of each variety were surface sterilized with $1 \% \mathrm{NaClO}$ for $15 \mathrm{~min}$, and then rinsed with distilled water. Thereafter, seeds were dried to remove excess water and allowed to dry at room temperature until their initial weight. After that, 50 seeds were sown on the moistened paper sheets in every germination box. And 10 boxes were sown for each variety, five of them suffered drought stress from the 10\% PEG in Hoagland solution, the rest five had no stress with Hoagland solution. Hoagland nutrient solution contained: macro elements $(\mathrm{mM}), 2.0 \mathrm{~K}_{2} \mathrm{SO}_{4}, 5.0 \mathrm{Ca}\left(\mathrm{NO}_{3}\right)_{2}, 0.50 \mathrm{Ca}\left(\mathrm{H}_{2} \mathrm{PO}_{4}\right)_{2}, 2.0$ $\mathrm{MgSO}_{4}$, and micro elements $(\mu \mathrm{M}) 40 \mathrm{FeSO}_{4}, 0.6 \mathrm{CuSO}_{4}, 1.6 \mathrm{ZnSO}_{4}, 10 \mathrm{MnCl}_{2}, 100 \mathrm{H}_{3} \mathrm{BO}_{3}, 0.2 \mathrm{H}_{2} \mathrm{MoO}_{4}$.

After sowing, germination boxes were placed in the growth chamber, where the temperature was maintained at $28^{\circ} \mathrm{C} / 22^{\circ} \mathrm{C}$ based on a $12: 12 \mathrm{~h}$ photoperiod $(4000 \mathrm{~lx})$. Each germination box was supplied the corresponding nutrient solution every day, and the $\mathrm{pH}$ of both solutions was maintained at $5.50 \pm 0.05$ by adding $1 \mathrm{~mol} \mathrm{~L}^{-1} \mathrm{HCl}$ or $1 \mathrm{~mol} \mathrm{~L}^{-1} \mathrm{NaOH}$ to the solutions during the germination and seedling stage.

\section{Sampling, data processing and analysis}

A seed was considered to be germinated when the 1-2 mm radicle appeared. Germination percentage was recorded each day until the $7^{\text {th }}$ day when seedlings were harvested. Ten seedlings were measured for root and shoot length. Mean germination time (MGT), germination index (GI), seedling vigor index (SVI) and mean seedling emergence time 
(MST) were calculated with the following Equations 1-4 (Zhang et al., 2013b):

$$
\begin{aligned}
\text { MGT } & =\sum(\mathrm{Dn}) / \Sigma \mathrm{n} \\
\mathrm{GI} & =\Sigma(\mathrm{D} / \mathrm{n})
\end{aligned}
$$

where $\mathrm{n}$ is the number of seeds germinated on day $\mathrm{D}$ (D representing the number of days since sowing).

$$
\begin{gathered}
\text { SVI }=\text { Percentage germination } \times \text { Seedling length }(\text { Shoot length }+ \text { Root length }) \\
\qquad \mathrm{MST}=\Sigma(\mathrm{Dm}) / \Sigma \mathrm{m}
\end{gathered}
$$

in the Equation 4, $\mathrm{m}$ is the number of seedling emerged on day $\mathrm{D}$ (D representing the number of days since sowing).

Drought tolerance coefficient (DTC) and membership function value of each comprehensive index of each genotype $\mathrm{U}\left(\mathrm{X}_{\mathrm{j}}\right)$ were obtained by the following Equations 5-8 in the statistical analysis (Toscano et al., 2017):

$$
\begin{aligned}
& \text { DTC }=\text { Drought stress character value/Control treatment character value } \\
& \qquad U\left(X_{j}\right)=\left(X_{j}-X_{\min }\right) /\left(X_{\max }-X_{\min }\right) \quad j=1,2, \ldots, n
\end{aligned}
$$

in the Equation $6, X_{j}$ represents the $j$-th comprehensive index of each genotype, $X_{\min }$ is the minimum value of $j$-th comprehensive index of each genotype, and $\mathrm{X}_{\max }$ is the maximum value of $\mathrm{j}$-th comprehensive index of each genotype.

$$
\mathrm{W}_{\mathrm{j}}=\mathrm{P}_{\mathrm{j}} / \sum_{\mathrm{j}=1}^{\mathrm{n}} \mathrm{Pj} \quad \mathrm{j}=1,2, \ldots, \mathrm{n}
$$

in the Equation $7, \mathrm{~W}_{\mathrm{j}}$ is the importance of the $\mathrm{j}$-th comprehensive indicator in all comprehensive indicators; $\mathrm{P}_{\mathrm{j}}$ is the contribution rate of the $j$-th comprehensive indicator of each genotype.

$$
D=\sum_{j=1}^{n}[U(X j) \times W j] \quad j=1,2, \ldots, n
$$

in the Equation 8, D value is the comprehensive evaluation value of drought tolerance of each genotype under the condition of drought stress during the germination stage of canola.

In this research, R software version 3.6.2 (R Foundation for Statistical Computing, Vienna, Austria) was used for statistical analyses, principal component analysis, regression analysis and cluster analysis. The differences between the means were compared by Fisher's protected least significant difference test $(\mathrm{P}<0.05)$.

\section{RESULTS}

\section{Drought tolerance coefficient of various characters in canola}

The drought tolerance coefficient of each single character was calculated by the results of each single character of the 53 canola varieties under drought stress and control treatments (Table 1). The average value of 14 single characteristic index under drought stress was lower than that of the control (except for the mean germination time (MGT), ratio of shoot and root dry weight (SRTD), ratio of shoot and root fresh weight (SRTF) of the 53 varieties during canola germination stage. Drought stress differently affected the indexes of germination-related characters of all varieties, MGT, germination index (GI), SRTF and root fresh weight per plant (RFW) changed greatly comparing the other 11 characters, and the changed degrees of the four characters were $14.7 \%-147.4 \%, 55.4 \%-8.9 \%, 49.5 \%-182.0 \%$ and $84.5 \%-43.1 \%$, respectively. The variation range of each character was different among canola varieties, so it was one-sided to evaluate the canola drought tolerance only according to the drought tolerance coefficient of a single character. Moreover, the correlation analysis showed that there was a large or small correlation between the drought tolerance coefficients of each character (Table 2), which made the information overlapped. At the same time, the contribution for each character greatly differed under drought stress. Therefore, these characters could not accurately evaluate the drought tolerance difference of each variety.

\section{Principal component analysis}

According to the principal component analysis (Table 3), contribution rates of the first six comprehensive indexes $\left(\mathrm{CI}_{1}, \mathrm{CI}_{2}, \mathrm{CI}_{3}, \mathrm{CI}_{4}, \mathrm{CI}_{5}\right.$ and $\left.\mathrm{CI}_{6}\right)$ were $32.3 \%, 17.5 \%, 12.3 \%, 10.7 \%, 8.2 \%$ and $6.5 \%$, respectively, and their cumulative contribution rate was $87.5 \%$. Consequently, the weak contributions from other index could be ignored compared with the first six comprehensive indexes. In this way, the original 16 single indicators were transformed into six new independent comprehensive indicators, representing $87.5 \%$ of the information of the original 16 single indicators. The first principal component was mainly determined by the six characters components, i.e., 1000-seeds weight (OSW), seedling dry weight 
Table 1. Contrast coefficients of morphological and physiological traits of canola varieties.

\begin{tabular}{|c|c|c|c|c|c|c|c|c|c|c|c|c|c|c|c|c|}
\hline Variety & MGT & GI & SVI & RL & SL & MST & RSTD & RSTF & RFW & SFW & RDW & SDW & FWPP & DWPP & OSW & SD \\
\hline ZYY8 & 1.02 & 1.02 & 0.94 & 0.95 & 1.06 & 0.83 & 1.03 & 1.51 & 0.41 & 0.63 & 0.81 & 0.84 & 0.61 & 0.83 & 1.08 & 1.12 \\
\hline YY57 & 0.86 & .08 & 0.83 & .80 & 0.85 & 0.80 & 1.13 & 1.66 & & 0.80 & & 0.71 & .77 & 0.69 & 0.53 & 0.84 \\
\hline YY817 & 0.87 & .08 & 0.76 & 0.74 & 0.81 & 0.78 & 0.99 & 1.23 & & 0.51 & 0.49 & 0.49 & 0.50 & 0.49 & 0.75 & .95 \\
\hline YY10 & 0.87 & .08 & 0.75 & 0.62 & 1.05 & 0.81 & 1.30 & 2.33 & & 1.37 & 0.92 & 1.20 & 1.29 & 1.14 & 1.17 & 1.14 \\
\hline ZY589 & 1.01 & 1.03 & 0.84 & 0.83 & 0.99 & 0.95 & 1.11 & 1.68 & 0.57 & 0.95 & 0.83 & 0.92 & 0.92 & 0.91 & 0.91 & 1.03 \\
\hline ZY28 & 0.94 & 1.05 & & 0.73 & & & 1.14 & 1.11 & & 0.52 & 0.44 & 0.50 & & 49 & 0.69 & .97 \\
\hline FY520 & 2.44 & 0.46 & 0.38 & 0.83 & 0.95 & 1.02 & 0.83 & & & 0.35 & 1.04 & 0.8 & 35 & 91 & 0.69 & 91 \\
\hline ZNY9 & 2.47 & 0.45 & 0.37 & 1.01 & 1.14 & 1.23 & 0.86 & 1.07 & 0.36 & 0.39 & 1.09 & 0.93 & 39 & 0.97 & 0.76 & 0.98 \\
\hline YG2009 & 1.05 & 1.00 & 0.93 & 0.95 & 1.05 & 0.87 & 0.84 & 1.42 & 0.45 & 0.64 & 0.79 & 0.66 & 0.62 & 0.69 & 0.69 & 0.94 \\
\hline YY50 & 0.93 & .05 & 0.87 & 0.82 & 1.02 & 0.85 & 1.29 & 1.66 & 0.55 & 0.91 & 0.88 & 1.13 & 0.88 & 1.08 & 0.99 & 1.05 \\
\hline ZYZ19 & 1.60 & 65 & 0.58 & 0.57 & 0.76 & 1.29 & 0.94 & 2.49 & 0.28 & 0.71 & 0.94 & 0.88 & 0.67 & 0.89 & 0.98 & 0.96 \\
\hline ZS12 & 1.01 & .03 & 0.78 & 0.77 & 0.91 & 0.87 & 1.01 & 1.16 & & 0.56 & 0.63 & 0.63 & & & 0.8 & 0.99 \\
\hline ZS9 & 1.09 & 0.97 & 0.95 & 0.99 & 1.00 & 0.95 & 0.65 & 1.30 & & 0.67 & 0.8 & 0.5 & & & 0. & 0.97 \\
\hline ZYZ781 & 0.96 & 1.03 & 1.12 & 1.19 & 0.93 & 0.88 & 0.83 & & & & & 0.7 & & & & 0.93 \\
\hline ZS11 & 1.03 & 0.9 & 0.93 & 0.93 & 0.9 & 0.95 & 0.94 & 1.02 & & & 0.96 & 0.8 & & & 0.9 & .98 \\
\hline CZY3 & 1.76 & 0.64 & 0.73 & 0.85 & 0.99 & 1.18 & 1.33 & 1.64 & & 0.5 & 0.3 & 0.50 & 5 & 47 & 0.51 & 0.84 \\
\hline FY792F1 & 0.91 & .06 & 0.72 & 0.64 & & 0.87 & 1.06 & 1.09 & & 0.6 & 0.6 & 0. & & & & 0.96 \\
\hline XZY814 & 0.91 & & 0. & & & 0.8 & 0.9 & & & & & & & & & 98 \\
\hline HXY12 & 1.01 & 98 & 0.88 & & & 0.9 & 0.7 & & & 0.6 & & & & & & 0.93 \\
\hline BYZ12 & 0.91 & 97 & 60 & 0 & 0.7 & & 0.89 & & & 0.5 & 0.8 & 0 . & & & & 0.93 \\
\hline JXY11 & 1.03 & 99 & 58 & 0.52 & 0.7 & 0.9 & 1.74 & & & 0.87 & 0.4 & & & 69 & & .98 \\
\hline CZY2 & 1.06 & 1.01 & 0.74 & 0.61 & 1.23 & 0.87 & 1.39 & 1.48 & & 0.84 & 0.69 & 0.9 & 2 & 90 & 22 & 92 \\
\hline CYZ83 & 1.21 & 0.85 & 0.74 & 0.78 & 0.81 & 1.15 & 1.10 & & & 0.65 & 0.63 & 0.6 & 64 & 0.67 & 0.69 & 0.93 \\
\hline XY9 & 1.20 & 94 & 0.69 & 0.72 & 0.85 & 1.11 & 1.62 & 0.7 & & 0.89 & 0.5 & 0.9 & 2 & 0.8 & 0.97 & 1.00 \\
\hline LY9 & 0.94 & .04 & 0.80 & 0.73 & 0.96 & 0.85 & 1.49 & 1.08 & & 1.23 & 0.7 & 1.1 & 1.2 & & 0.9 & 0.97 \\
\hline QY6 & 1.44 & 78 & 0.84 & 0.94 & 0.98 & 1.22 & 1.47 & 0.91 & & 0.80 & 0.5 & 0.8 & 0.8 & 0.77 & 0.7 & 0.94 \\
\hline RHY6 & 1.40 & 77 & 0.69 & 0.70 & 0.91 & 1.15 & 1.3 & & & 0.71 & 0.5 & 0.6 & & & & 0.99 \\
\hline KLY1 & 1.08 & 0.89 & 0.79 & 0.72 & & 1.26 & 2.26 & 2.82 & & & & 0.8 & & & & 1.01 \\
\hline BY517 & 0.92 & 1.06 & 0.69 & 0.69 & & 0.85 & & & & & 0.6 & 0.5 & & & & 0.95 \\
\hline GYZ6 & 0.87 & 1.06 & 0.87 & 0.91 & & 1.01 & & & & & 0.5 & 0.6 & & & 0. & 0.93 \\
\hline CJZY3 & 0.85 & 1.09 & 0.89 & 0.84 & & & & & & & & 0.8 & & & & 1.00 \\
\hline QDJ & 0 & 1.05 & 0.9 & 0.9 & 1.0 & 0.9 & 1.2 & & & 0.6 & 0.5 & 0. & & & 0. & 1.03 \\
\hline CSY & 0.9 & 1.01 & 1.09 & 1.10 & 1.0 & 1.0 & 0.5 & 1.4 & & 0.5 & 1.3 & 0.7 & 48 & 0 & 0. & 1.02 \\
\hline JLY5 & 0 & 1.05 & 0.80 & 0.77 & 0.8 & 0.8 & 0.51 & 0.6 & & 0.6 & 1.2 & 0.6 & 64 & 0 & 0.80 & 1.00 \\
\hline JLY & 0 & .04 & 0.88 & 0.87 & 0.8 & 0 & 1.32 & & & 0.6 & 0.6 & 0.8 & 71 & 0 & 0. & 1.00 \\
\hline JLY10 & 0.87 & 08 & 0.86 & 0.84 & 0.8 & 0.7 & 0.90 & & & 0.8 & 0.6 & 0.6 & 7 & 0 & 0.79 & 0.98 \\
\hline YBS & 0.92 & .06 & 0.90 & 0.85 & 1.03 & 0.92 & 0.77 & 1.81 & 0.52 & 0.94 & 1.00 & 0.77 & 0.90 & 0.82 & 0.88 & 1.00 \\
\hline JLYT & 0.96 & 1.01 & 0.84 & 0.82 & 0.82 & 1.03 & 0.57 & 0.71 & & 1.02 & 1.52 & 0.87 & 1.06 & 1.00 & 0.98 & 1.06 \\
\hline YB & 1.14 & & 0.99 & 1.05 & 0.92 & 1.03 & 0.74 & & & 1.01 & 0.94 & 0.70 & 1.04 & & 0.92 & 1.04 \\
\hline DAZ600 & 1.12 & 93 & 0.75 & 0.74 & 0.86 & 1.10 & 0.93 & 1.15 & 0.40 & 0.46 & 1.09 & 1.01 & 0.45 & 1.03 & 0.90 & 1.03 \\
\hline XDZY9 & 0.88 & 1.08 & 0.87 & 0.88 & 0.83 & 0.93 & 1.10 & 0.94 & & 0.83 & 0.54 & 0.60 & 0.83 & 0.59 & 0.91 & 0.98 \\
\hline DZY10 & 1.34 & & 0.51 & 0.79 & 0.91 & 1.22 & 1.01 & 1.26 & & & 0.98 & 0.99 & 0.51 & 0.99 & 0.81 & 0.96 \\
\hline JHH & 0.87 & & 0.66 & 0.58 & & & & 1.11 & & 0.67 & 0.4 & 0.38 & 0.66 & 0.39 & 0.72 & 0.90 \\
\hline DZY5 & 1.24 & 0.86 & 0.50 & 0.45 & & 1.0 & 2.2 & 1.2 & & 0.50 & 0.38 & 0.85 & 0.49 & 0.75 & 0.82 & 0.98 \\
\hline XY50 & 1.06 & 1.01 & 0.72 & 0.76 & & & & 0.6 & & 0.6 & 1.1 & 0.4 & 0.69 & 0.60 & 0.7 & 0.96 \\
\hline & & & & & & & & & & & & & & & & 0.92 \\
\hline ZY18 & 1.11 & & & & & & & & & & & & & & & 0.96 \\
\hline YY9 & 0.92 & 1.06 & 0.78 & 0.75 & 0.8 & & 0.86 & 1.50 & & 0.7 & 0.7 & 0.68 & 0.70 & 0.71 & 0.90 & 0.92 \\
\hline BDHZ & 1.20 & 0.93 & 0.44 & 0.39 & 0.71 & 0.90 & 0.49 & 1.10 & 0.4 & 0.5 & 1.1 & 0.55 & & 0.67 & 0.65 & 0.88 \\
\hline FYJ & 0.99 & 1.02 & 0.84 & 0.85 & 0.87 & 0.84 & 0.90 & 1.38 & & 0.6 & 0.7 & 0.67 & 0.59 & 0.69 & 0.82 & 0.97 \\
\hline YBA & 0.95 & 0.83 & 0.82 & 0.82 & 1.07 & 1.14 & 1.20 & 2.13 & 0.26 & 0.55 & 0.63 & 0.75 & 0.52 & 0.72 & 1.00 & 1.01 \\
\hline QY20 & 1.06 & 1.01 & 0.70 & 0.73 & 0.68 & 1.00 & 1.25 & 2.25 & 0.59 & 1.34 & 0.63 & 0.78 & 1.27 & 0.75 & 0.84 & 1.02 \\
\hline QZ7 & 1.05 & 1.03 & 0.77 & 0.81 & 0.74 & 0.86 & 1.47 & 2.31 & 0.39 & 0.90 & 0.69 & 1.01 & 0.85 & 0.94 & 0.83 & 0.99 \\
\hline
\end{tabular}

MGT: Mean germination time; GI: germination index; SVI: seedling vigor index; RL: root length; SL: shoot length; MST: mean seedling emergence time; RSTD: ratio of shoot and root dry weight; RSTF: ratio of shoot and root fresh weight; RFW: root fresh weight per plant; SFW: shoot fresh weight per plant; RDW: root dry weight per plant; SDW: shoot dry weight per plant; FWPP: fresh weight per plant; DWPP: dry weight per plant; OSW: 1000-seeds weight; SD: seed diameter. 
Table 2. Correlation matrix of drought-tolerance coefficients of different characters.

\begin{tabular}{|c|c|c|c|c|c|c|c|c|c|c|c|c|c|c|}
\hline Index & DWPP & FWPP & GI & MGT & MST & OSW & RDW & RFW & RL & RSTD & RSTF & SD & SDW & SFW \\
\hline FWPP & $0.439^{* * *}$ & & & & & & & & & & & & & \\
\hline GI & 0.031 & $0.359^{* *}$ & & & & & & & & & & & & \\
\hline MGT & $0.293^{*}$ & -0.038 & $-0.661^{* *}$ & & & & & & & & & & & \\
\hline MST & $0.287^{*}$ & -0.082 & $-0.397^{* *}$ & $0.535^{* *}$ & & & & & & & & & & \\
\hline OSW & $0.640^{* * *}$ & $0.509^{* *}$ & $0.361^{* *}$ & -0.040 & 0.158 & & & & & & & & & \\
\hline RDW & $0.610^{* * *}$ & 0.234 & 0.019 & $0.315^{*}$ & 0.074 & $0.340^{*}$ & & & & & & & & \\
\hline RFW & 0.147 & $0.656^{* *}$ & 0.211 & 0.081 & -0.061 & 0.245 & $0.285^{*}$ & & & & & & & \\
\hline RL & 0.253 & 0.220 & 0.110 & 0.254 & 0.231 & $0.270^{*}$ & $0.364^{* *}$ & $0.343^{* * *}$ & & & & & & \\
\hline RSTD & 0.246 & $0.294^{*}$ & -0.002 & 0.258 & 0.243 & 0.220 & $-0.331^{*}$ & 0.136 & -0.030 & & & & & \\
\hline RSTF & $0.360^{* * *}$ & 0.230 & 0.043 & 0.189 & 0.135 & $0.302^{*}$ & 0.097 & $-0.338^{*}$ & -0.027 & $0.494^{* *}$ & & & & \\
\hline SD & $0.330^{*}$ & 0.156 & $0.398^{* *}$ & -0.141 & $0.371^{* *}$ & $0.585^{* *}$ & 0.063 & -0.025 & 0.172 & -0.050 & -0.031 & & & \\
\hline SDW & $0.957^{* *}$ & $0.474^{* *}$ & 0.015 & $0.293^{*}$ & $0.293^{*}$ & $0.628^{* *}$ & $0.387^{* *}$ & 0.129 & 0.192 & $0.482^{* *}$ & $0.446^{* *}$ & $0.283^{*}$ & & \\
\hline SFW & $0.462^{* *}$ & $0.994^{* *}$ & $0.346^{* *}$ & -0.017 & -0.084 & $0.513^{* *}$ & 0.237 & $0.583^{* *}$ & 0.203 & $0.327^{*}$ & $0.326^{*}$ & 0.133 & $0.505^{* *}$ & \\
\hline SL & $0.432^{* *}$ & 0.209 & 0.061 & $0.282^{*}$ & $0.326^{*}$ & $0.487^{* * *}$ & 0.225 & 0.087 & 0.565 & 0.103 & 0.149 & $0.428^{* *}$ & $0.420^{* *}$ & 0.212 \\
\hline SVI & 0.147 & $0.422^{* *}$ & $0.589^{* *}$ & $-0.286^{*}$ & -0.049 & $0.427^{* *}$ & 0.198 & $0.393^{* *}$ & $0.743^{* *}$ & 0.062 & 0.086 & 0.247 & 0.126 & $0.407^{* *}$ \\
\hline
\end{tabular}

*,**Significant at 0.05 and 0.01 level.

DWPP: Dry weight per plant; FWPP: fresh weight per plant; GI: germination index; MGT: mean germination time; MST: mean seedling emergence time; OSW: 1000-seeds weight; RDW: root dry weight per plant; RFW: root fresh weight per plant; RL: root length; RSTD: ratio of shoot and root dry weight; RSTF: ratio of shoot and root fresh weight; SD: seed diameter; SDW: shoot dry weight per plant; SFW: shoot fresh weight per plant; SL: shoot length; SVI: seedling vigor index.

per plant (DWPP), shoot dry weight (SDW), shoot fresh weight (SFW), seedling fresh weight per plant (FWPP), and shoot length (SL), which could be summarized as biomass character factors. The second principal component was determined by MGT, GI, mean seedling emergence time (MST), seedling vigor index (SVI), SDW and root fresh weight per plant (RFW), which could be summarized as biomass factors and germination time factors. The third, fourth, fifth and sixth principal components were determined by root length (RL), ratio of shoot and root dry weight (RSTD), average RFW, seed diameter (SD) and ratio of shoot and root fresh weight (RSTF), which could be roughly categorized as biomass factors and quantitative factors.

\section{Comprehensive evaluation and cluster analysis of drought tolerance coefficient}

The membership function value was calculated by the Equation 6 for all representative indexes of each variety. Equation 7 was used to calculate the weight of six representative indexes. The comprehensive evaluation D-value of drought resistance could be obtained according to the U-value and weight of each representative index of each variety by Equation 8 (Table 4). The drought tolerance could be evaluated comparing the D-value of each variety. In this research, such four varieties with higher D-value had higher drought resistance as YY10 (0.790), LY9 (0.701), JLYT (0.684) ZS11 (0.668) and YY50 (0.658). Euclidean distance and maximum distance method were used to cluster D-value to evaluate varieties drought tolerance difference comprehensively (Figure 1), and in this way, the 53 varieties could be classified into three categories. The first-class varieties with the strongest drought resistance were YY10, JLYT, LY9, ZS11, and YY50; there were 16 varieties belonging to the second one with moderate drought resistance, such as QZ7, DAZ600, ZYZ19 and so on; and the third category was the rest 32 varieties with the weakest drought resistance, which were BY517, JHH, CZY3 and so on.

Table 3. Coefficients of comprehensive index (CI) and their contribution rate (CR).

\begin{tabular}{|c|c|c|c|c|c|c|c|c|c|c|c|c|c|c|c|c|c|}
\hline $\begin{array}{l}\text { Comprehensi } \\
\text { index }\end{array}$ & Ie DWPP & FWPP & GI & MGT & MST & OSW & RDW & RFW & RL & RSTD & RSTF & SD & SDW & SFW & SL & SVI & $\begin{array}{l}\mathrm{CR} \\
(\%)\end{array}$ \\
\hline $\mathrm{I}_{4}$ & -0.3493 & -0.3332 & -0.1468 & -0.0772 & -0.1081 & -0.3543 & -0.2139 & -0.205 & -0.2324 & -0.1562 & -0.165 & -0.1965 & -0.3468 & -0.336 & -0.2669 & -0.2565 & 32.3 \\
\hline $\mathrm{Cl}_{2}$ & -0.2148 & 0.1925 & 0.464 & -0.4807 & -0.3935 & 0.0364 & -0.0818 & 0.2187 & 0.0303 & -0.1672 & -0.1955 & & -0.2319 & 0.1662 & -0.1138 & 0.3104 & 17.5 \\
\hline $\mathrm{Cl}_{3}$ & 3363 & 0.2523 & 0.0345 & -0.0888 & -0.2003 & -0.0098 & -0.2456 & -0.0259 & -0.439 & 0.4292 & 0.3554 & -0.2391 & 0.1681 & 0.2878 & -0.3157 & -0.2 & 12.3 \\
\hline $\mathrm{Cl}_{4}$ & 0.0457 & -0.2319 & 0.2736 & -0.3137 & 0.1075 & 0.2424 & -0.255 & -0.5064 & -0.1654 & 0.0562 & 0.2281 & 0.4755 & 0.0827 & -0.2047 & 0.1453 & 0.0241 & 10.7 \\
\hline $\mathrm{Cl}_{5}$ & -0.3441 & 0.0163 & -0.0281 & 0.1158 & 0.2565 & -0.1284 & -0.5316 & 0.111 & 0.3018 & 0.4788 & 0.0545 & -0.0464 & -0.1769 & 0.0096 & 0.2042 & 0.3023 & 8.2 \\
\hline $\mathrm{Cl}_{6}$ & -0.0079 & 0.1526 & -0.0711 & 0.0008 & 0.3184 & 0.1182 & -0.2338 & 0.3438 & -0.2981 & 0.0401 & -0.5292 & 0.4453 & 0.0174 & 0.0805 & -0.0887 & -0.316 & 6.5 \\
\hline
\end{tabular}

DWPP: Dry weight per plant; FWPP: fresh weight per plant; GI: germination index; MGT: mean germination time; MST: mean seedling emergence time; OSW: 1000-seeds weight; RDW: root dry weight per plant; RFW: root fresh weight per plant; RL: root length; RSTD: ratio of shoot and root dry weight; RSTF: ratio of shoot and root fresh weight; SD: seed diameter; SDW: shoot dry weight per plant; SFW: shoot fresh weight per plant; SL: shoot length; SVI: seedling vigor index. 
Table 4. The value of comprehensive index (CI), index weight (IW), membership function (U(X)), evaluation (D) and prediction $(P)$ of drought tolerance of each variety.

\begin{tabular}{|c|c|c|c|c|c|c|c|c|c|c|c|c|c|c|c|}
\hline Varieties & $\mathrm{CI}_{1}$ & $\mathrm{CI}_{2}$ & $\mathrm{CI}_{3}$ & $\mathrm{CI}_{4}$ & $\mathrm{CI}_{5}$ & $\mathrm{CI}_{6}$ & $\mathrm{U}\left(\mathrm{X}_{1}\right)$ & $\mathrm{U}\left(\mathrm{X}_{2}\right)$ & $\mathrm{U}\left(\mathrm{X}_{3}\right)$ & $\mathrm{U}\left(\mathrm{X}_{4}\right)$ & $\mathrm{U}\left(\mathrm{X}_{5}\right)$ & $\mathrm{U}\left(\mathrm{X}_{6}\right)$ & $\mathrm{P}$ & D & Rank \\
\hline ZYY8 & 45.592 & 34.910 & 46.317 & 28.850 & 30.702 & 35.630 & 0.591 & 0.274 & 0.896 & 0.104 & 0.097 & 0.865 & 0.142 & 0.485 & 20 \\
\hline YY57 & 48.259 & 36.547 & 48.183 & 31.933 & 26.951 & 35.513 & 0.401 & 0.449 & 0.991 & 0.006 & 0.039 & 0.028 & -0.009 & 0.384 & 39 \\
\hline YY817 & 35.933 & 28.487 & 36.968 & 23.712 & 23.423 & 28.917 & 0.134 & 0.164 & 0.992 & 0.013 & 0.011 & 0.356 & -0.087 & 0.251 & 52 \\
\hline YY10 & 69.759 & 49.833 & 64.864 & 46.906 & 29.333 & 43.021 & 1.000 & 1.000 & 0.987 & 0.008 & 0.069 & 1.000 & 0.292 & 0.790 & 1 \\
\hline ZY589 & 55.771 & 41.061 & 54.315 & 36.872 & 29.589 & 38.763 & 0.689 & 0.604 & 0.903 & 0.096 & 0.331 & 0.606 & 0.180 & 0.590 & 8 \\
\hline ZY28 & 35.817 & 28.262 & 36.717 & 23.823 & 22.958 & 28.491 & 0.130 & 0.176 & 0.939 & 0.055 & 0.178 & 0.272 & -0.074 & 0.259 & 51 \\
\hline FY520 & 30.314 & 21.560 & 31.242 & 19.714 & 22.938 & 22.459 & 0.689 & 0.000 & 0.017 & 0.981 & 0.474 & 0.273 & 0.275 & 0.441 & 26 \\
\hline ZNY9 & 33.629 & 23.126 & 35.346 & 22.065 & 25.400 & 24.919 & 0.771 & 0.043 & 0.000 & 1.000 & 0.882 & 0.382 & 0.354 & 0.526 & 16 \\
\hline YG2009 & 44.767 & 34.296 & 46.184 & 28.799 & 29.562 & 35.547 & 0.401 & 0.290 & 0.859 & 0.123 & 0.183 & 0.267 & 0.036 & 0.379 & 41 \\
\hline YY50 & 55.894 & 41.463 & 53.719 & 36.141 & 31.065 & 38.468 & 0.919 & 0.563 & 0.946 & 0.050 & 0.136 & 0.729 & 0.252 & 0.658 & 5 \\
\hline ZYZ19 & 42.692 & 31.689 & 41.416 & 28.075 & 23.420 & 30.100 & 0.672 & 0.339 & 0.320 & 0.463 & 1.000 & 0.718 & 0.288 & 0.564 & 11 \\
\hline ZS12 & 39.326 & 30.544 & 39.796 & 25.744 & 25.368 & 30.508 & 0.320 & 0.219 & 0.907 & 0.094 & 0.182 & 0.460 & 0.017 & 0.352 & 46 \\
\hline ZS9 & 45.915 & 34.863 & 47.444 & 29.950 & 29.753 & 36.675 & 0.320 & 0.329 & 0.815 & 0.148 & 0.349 & 0.668 & 0.054 & 0.399 & 38 \\
\hline ZYZ781 & 51.262 & 38.911 & 52.851 & 32.968 & 34.314 & 41.263 & 0.493 & 0.399 & 0.916 & 0.068 & 0.202 & 0.470 & 0.081 & 0.453 & 22 \\
\hline ZS11 & 61.095 & 44.891 & 59.211 & 40.430 & 33.988 & 43.250 & 0.816 & 0.691 & 0.862 & 0.091 & 0.437 & 0.742 & 0.247 & 0.668 & 4 \\
\hline CZY3 & 38.100 & 28.727 & 40.675 & 25.052 & 24.560 & 30.679 & 0.113 & 0.210 & 0.306 & 0.558 & 0.797 & 0.000 & 0.017 & 0.270 & 50 \\
\hline FY792F1 & 42.863 & 32.816 & 41.480 & 28.582 & 24.448 & 30.617 & 0.441 & 0.342 & 0.958 & 0.036 & 0.186 & 0.580 & 0.064 & 0.431 & 28 \\
\hline XZY814 & 33.207 & 26.628 & 32.680 & 21.426 & 22.112 & 25.324 & 0.435 & 0.091 & & 0.036 & & 0.426 & 0.046 & & 43 \\
\hline HXY12 & 44.615 & 34.140 & 45.337 & 28.905 & 28.30 & 34.741 & 0.447 & & & 0.099 & & & & & 34 \\
\hline BYZ12 & 34.997 & 27.631 & 33.967 & 22.792 & 21.94 & 25.831 & 0.551 & & & 36 & & & 0.083 & & 33 \\
\hline JXY11 & 47.128 & 35.007 & 45.296 & 32.122 & 21.878 & 31.361 & & & & 0.109 & 80 & & & & 24 \\
\hline CZY2 & 50.662 & 37.800 & 48.335 & 33.306 & 26.873 & 33.879 & & & & 0.125 & & & & & 13 \\
\hline CYZ83 & 41.981 & 31.777 & 42.034 & 27.727 & 25.344 & 31.440 & 0.378 & 0.309 & 0.624 & 0.218 & 0.721 & 0.263 & 0.089 & 0.403 & 37 \\
\hline SXY9 & 52.690 & 38.738 & 49.625 & 36.342 & 26.700 & 35.229 & 0.643 & 0.606 & 0.765 & 0.216 & 0.652 & 0.688 & 0.213 & 0.605 & 6 \\
\hline LY9 & 66.166 & 47.678 & 61.538 & 45.104 & 29.909 & 41.923 & 0.873 & 0.922 & 0.928 & 0.052 & 0.135 & 0.595 & 0.225 & 0.701 & 2 \\
\hline QY6 & 50.753 & 37.418 & 50.559 & 33.767 & 29.555 & 37.027 & 0.510 & 0.488 & 0.517 & 0.364 & 0.875 & 0.319 & 0.175 & 0.509 & 17 \\
\hline RHY6 & 43.610 & 32.550 & 42.951 & 29.533 & 24.331 & 31.318 & 0.320 & 0.386 & 0.511 & 0.339 & 0.724 & 0.386 & 0.091 & 0.405 & 36 \\
\hline KLY1 & 36.249 & 29.285 & 37.839 & 22.333 & 26.558 & 30.203 & 0.533 & 0.068 & 0.695 & 0.141 & 0.951 & 0.447 & 0.173 & 0.448 & 25 \\
\hline BY517 & 33.884 & 26.993 & 34.596 & 22.234 & 22.511 & 27.213 & 0.257 & 0.115 & 0.954 & 0.041 & 0.150 & 0.262 & -0.031 & 0.291 & 49 \\
\hline GYZ6 & 36.555 & 29.278 & 37.713 & 23.427 & 27.068 & 30.572 & 0.332 & 0.115 & 0.961 & 0.013 & 0.449 & 0.270 & 0.022 & 0.345 & 48 \\
\hline CJZY3 & 49.908 & 37.795 & 49.645 & 32.320 & 29.209 & 36.865 & 0.562 & 0.430 & 1.000 & 0.000 & 0.000 & 0.904 & 0.112 & 0.501 & 19 \\
\hline QDJ & 46.634 & 36.036 & 46.674 & 31.875 & 29.348 & 36.817 & 0.262 & 0.388 & 0.947 & 0.041 & 0.295 & 0.496 & 0.002 & 0.377 & 42 \\
\hline CSY & 43.222 & 33.952 & 45.238 & 26.311 & 34.113 & 36.988 & 0.677 & 0.142 & 0.872 & 0.065 & 0.527 & 0.658 & 0.195 & 0.5 & 18 \\
\hline JLY5 & 42.472 & 32.737 & 41.369 & 28.650 & 26.882 & 32.231 & 0.504 & 0.305 & 0.945 & 0.036 & 0.169 & 0.438 & 0.076 & 0.433 & 27 \\
\hline JLY7 & 47.008 & 35.820 & 46.382 & 30.925 & 28.967 & 35.074 & 0.527 & 0.380 & & 0.057 & 0.086 & 0.50 & 0.084 & & 23 \\
\hline JLY10 & 51.423 & 38.434 & 50.563 & & 27.4 & & & & & 0.010 & & & -0.009 & & 35 \\
\hline YBS & 55.086 & 40.855 & 54.3 & & & & & & & & & & & & 15 \\
\hline JLYT & 59.920 & 43.797 & 56.133 & & & & & & & & & 0.71 & 0.249 & & 3 \\
\hline YB & 60.342 & 44.133 & 59.273 & 41.598 & 32.537 & 44.186 & 0.476 & 0.736 & 0.750 & 0.180 & 0.488 & 0.625 & 0.126 & 0.542 & 14 \\
\hline DAZ600 & 37.849 & 29.606 & 36.843 & 23.337 & 27.462 & 28.558 & 0.853 & 0.112 & 0.747 & 0.164 & 0.635 & 0.582 & 0.277 & 0.565 & 10 \\
\hline XDZY9 & 49.995 & 37.532 & 49.712 & 34.081 & 27.530 & 37.051 & 0.262 & 0.513 & 0.980 & 0.019 & 0.303 & 0.610 & 0.009 & 0.414 & 31 \\
\hline DZY10 & 37.997 & 27.874 & 37.444 & 24.733 & 24.936 & 26.949 & 0.798 & 0.174 & 0.534 & 0.302 & 0.858 & 0.451 & 0.284 & 0.556 & 12 \\
\hline JHH & 39.356 & 30.321 & 39.059 & 27.356 & 20.567 & 28.919 & 0.000 & 0.336 & 0.982 & 0.013 & 0.007 & 0.314 & -0.140 & 0.231 & 53 \\
\hline DZY5 & 33.446 & 26.094 & 31.855 & 21.940 & 19.829 & 22.930 & 0.481 & 0.157 & 0.643 & 0.237 & 0.518 & 0.466 & 0.126 & 0.412 & 32 \\
\hline XY50 & 42.614 & 32.223 & 41.900 & 29.787 & 24.685 & 31.967 & 0.285 & 0.367 & 0.873 & 0.128 & 0.087 & 0.383 & -0.007 & 0.354 & 45 \\
\hline ZYZ108 & 46.397 & 34.866 & 47.003 & 29.862 & 28.589 & 35.016 & 0.487 & 0.354 & 0.554 & 0.308 & 0.757 & 0.598 & 0.169 & 0.482 & 21 \\
\hline ZY18 & 45.892 & 34.663 & 44.753 & 32.504 & 25.387 & 33.987 & 0.228 & 0.456 & 0.799 & 0.161 & 0.211 & 0.445 & -0.008 & 0.360 & 44 \\
\hline YY9 & 45.214 & 34.317 & 44.763 & 29.962 & 25.996 & 33.316 & 0.424 & 0.376 & 0.957 & 0.039 & 0.074 & 0.589 & 0.049 & 0.422 & 29 \\
\hline BDHZ & 33.186 & 25.524 & 31.163 & 23.016 & 18.014 & & 0.378 & & & & & & 0.040 & 0.350 & 47 \\
\hline FYJ & 42.006 & 32.382 & 42.671 & 27.214 & & & & & & & & & 0.042 & 0.382 & 40 \\
\hline YBA & 40.420 & 31.038 & 41.834 & & & & & & & 0.062 & 0.706 & 0.748 & 0.131 & 0.416 & 30 \\
\hline QY20 & 65.111 & 46.323 & 62.547 & 45.132 & 25.958 & 42.093 & 0.481 & & 0.882 & 0.125 & 0.446 & 0.504 & 0.109 & 0.591 & 7 \\
\hline QZ7 & 52.718 & 39.106 & 51.628 & 34.637 & 28.256 & 37.111 & 0.741 & 0.532 & 0.916 & 0.125 & 0.156 & 0.477 & 0.177 & 0.574 & 9 \\
\hline IW & & & & & & & 36.9 & 20.0 & 14.1 & 12.2 & 9.4 & 7.4 & & & \\
\hline
\end{tabular}

P: Prediction value of drought tolerance; D: comprehensive evaluation value of drought tolerance; $\mathrm{CI}_{1} \sim \mathrm{CI}_{6}$ : value of first $\left(\mathrm{CI}_{1}\right)$, second $\left(\mathrm{CI}_{2}\right)$, third $\left(\mathrm{CI}_{3}\right)$, fourth $\left(\mathrm{Cl}_{4}\right)$, fifth $\left(\mathrm{CI}_{5}\right)$ and sixth $\left(\mathrm{CI}_{6}\right)$ comprehensive index, respectively; $\mathrm{U}\left(\mathrm{X}_{1}\right) \sim \mathrm{U}\left(\mathrm{X}_{6}\right)$ : value of the membership function of first $\left(\mathrm{U}\left(\mathrm{X}_{1}\right)\right)$, second $\left(\mathrm{U}\left(\mathrm{X}_{2}\right)\right)$, third $\left(\mathrm{U}\left(\mathrm{X}_{3}\right)\right)$, fourth $\left(\mathrm{U}\left(\mathrm{X}_{4}\right)\right)$, fifth $\left(\mathrm{U}\left(\mathrm{X}_{5}\right)\right)$ and sixth $\left(\mathrm{U}\left(\mathrm{X}_{6}\right)\right)$ comprehensive index, respectively. 
Figure 1. Dendrogram based on D value by cluster analysis.

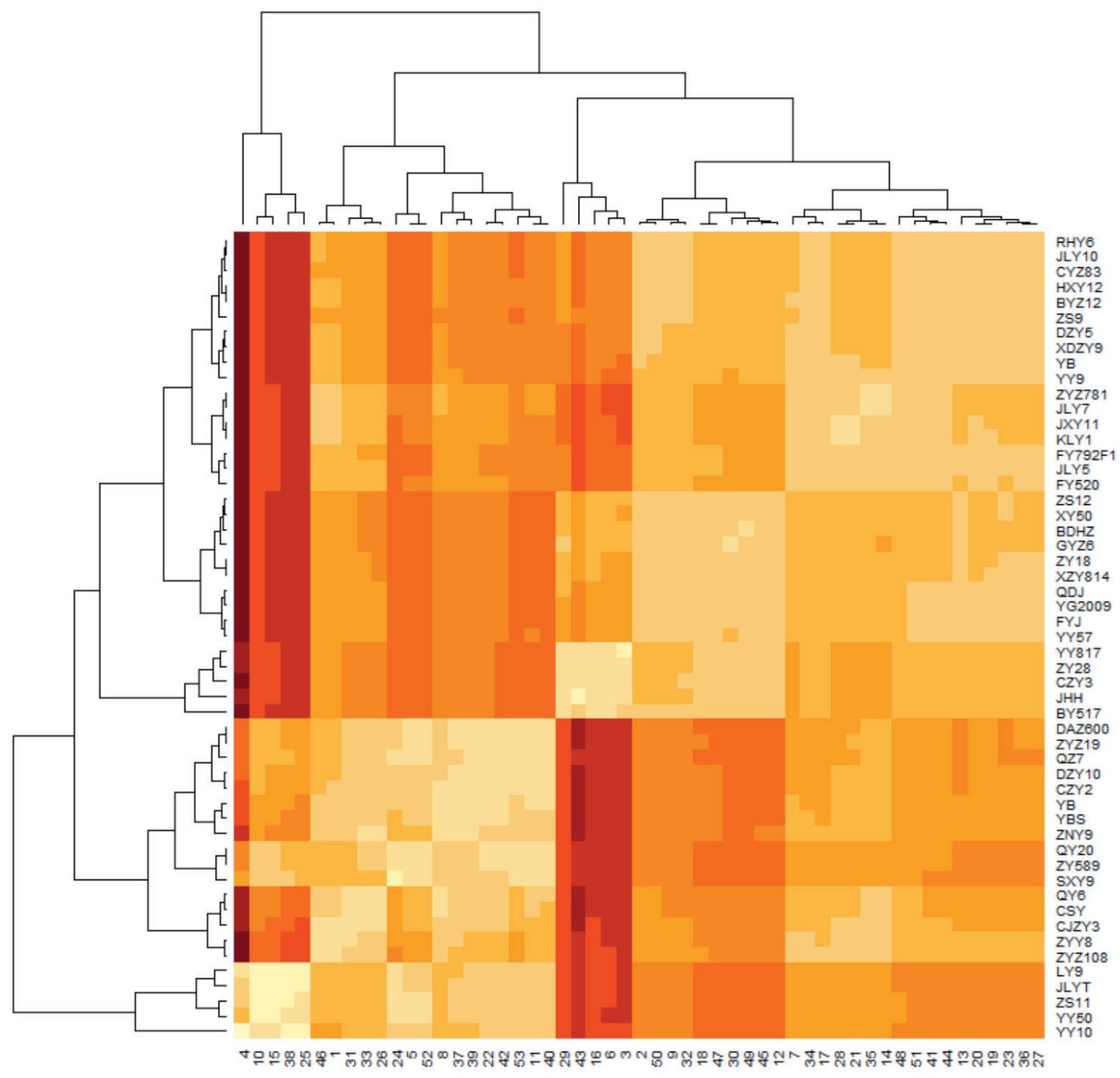

Rescaled distance cluster combine.

\section{Screening indices and drought tolerance coefficient in canola}

The mathematical model evaluating drought resistance was established and predicted the drought resistance. The stepwise regression analysis of 16 characteristic indicators was carried out to screen the drought resistance indicators of different canola varieties during germination and emergence stage. The optimal regression equation was established, and took the comprehensive evaluation value of drought tolerance ( $\mathrm{D}$-value) as the dependent variable and the drought tolerance coefficient of each single indicator as the independent variable: $\mathrm{D}=-0.601+0.225 \mathrm{X}_{1}+0.105 \mathrm{X}_{2}+0.207 \mathrm{X}_{3}+0.182 \mathrm{X}_{5}+$ $0.110 \mathrm{X}_{6}+0.036 \mathrm{X}_{13}\left(\mathrm{~F}=23836.39^{* *}, \mathrm{R}^{2}=0.9999\right)$, and in this formula, $\mathrm{X}_{1}, \mathrm{X}_{2}, \mathrm{X}_{3}, \mathrm{X}_{5}, \mathrm{X}_{6}$ and $\mathrm{X}_{13}$ represented DWPP, FWPP, GI, MST, OSW and SDW, respectively. It would be inferred from the equation that among the 16 single indicators, the above six indicators significantly influenced drought tolerance of canola seedlings at germination period. Therefore, these six indexes could be determined selectively to evaluate difference in drought tolerance. The prediction value of drought tolerance $(\mathrm{P})$ was significantly correlated with the comprehensive evaluation value of drought tolerance (D-value) for the 53 canola varieties $\left(r=0.8399^{* *}\right)$, which effectively proved the equation accuracy in predicting varieties drought tolerance difference. 


\section{DISCUSSION}

\section{Selecting drought tolerance characters and identifying canola varieties with drought tolerance during germination and emergence stages}

Crop drought tolerance identification was a procedure identifying, screening, evaluating and classifying different varieties with varied drought tolerance capacity (Li et al., 2010; Bai et al., 2014; Channaoui et al., 2019). The most important step was to select clear and effective identification indices of drought-resistant varieties in a much earlier growing period (Xie et al., 2013). Some research showed that there could be a single physiological indicator for drought resistance identification in canola, such as crop canopy temperature difference, detached leaves dehydration rate, leaves water potential and osmotic potential, pod osmotic adjustment ability, chlorophyll stability in leaves, germination rate under low osmotic condition and relative vigor index (Li et al., 2010; Xie et al., 2013; Bai et al., 2014; Li et al., 2015; Channaoui et al., 2019).

However, crop drought tolerance was a quantitative genetic trait controlled by multiple genes (Byzova et al., 2004; Jian et al., 2016). Different varieties owned different traits related to drought tolerance, which was affected by certain genes and growing environment (Byzova et al., 2004; Muller et al., 2005; Zhao et al., 2005; Li et al., 2010; Zhang et al., 2013a; Jian et al., 2016). It was difficult to evaluate drought tolerance comprehensively and accurately through a single index. So, the multi-index membership function method was advised to evaluate drought tolerance in some crops. Although it would reflect many drought-tolerance characters of crops relatively comprehensively, the membership function showed much certain limitation.

In recent years, the comprehensive evaluation had already been preliminarily applied to identify drought-resistant germplasm, such as wheat, corn, soybean and other crops (Khodarahmpour and Motamedi, 2011; Ali et al., 2018; Farid and Ridwan, 2018; Liu et al., 2019b; Pavia et al., 2019). The comprehensive evaluation was involved in the principal component analysis, membership function analysis, regression analysis and cluster analysis of drought tolerance indices correlation for different varieties. In this study, 16 indicators including morphological and agronomic characters were selected in germination and emergence stages when canola was most prone to drought damage. These 16 single indicators were synthesized into six independent comprehensive indexes to identify drought tolerance difference among the studied 53 varieties with principal component analysis. The obtained six indexes were screened by stepwise regression analysis, and regression equation was established. Lastly, these 53 canola varieties could be classified in the drought tolerance difference by cluster analysis.

\section{Drought tolerance characteristics during canola germination and emergence stages}

Winter canola greatly required water during the whole growing season, and its adaptability was poor to drought stress (Hamzei and Soltani, 2012; Shabani et al., 2014). Drought stress would affect root development, plant growth, and ultimately lead to a significant decline in yield and yield components (Marcinkeviciene et al., 2013; Shabani et al., 2014). The variety with strong drought tolerance would have a stronger adaptability to arid environment, which could significantly reduce water transpiration and improve the capacity of osmoregulation substance metabolism in the case of drought stress (Borisjuk et al., 2013; Kuai et al., 2016).

In this experiment, 1000-seeds weight, fresh and dry seedling weight, germination time and some root characters occupied the first, second and third principal component positions respectively. The results showed that DM accumulation, germination time and root characters were the important performance indexes related to drought tolerance among different canola varieties under some drought conditions (Xie et al., 2013; Channaoui et al., 2019). Under drought conditions, some excellent characters' performance in canola germination and emergence stages might be benefit for obtaining more nutrients, water, light and other resources in the growing environment; and the performance could contribute to the competition ability of the individual seedling (Borisjuk et al., 2013; Bai et al., 2014).

However, the effects of drought stress on seedlings were not only performed in the phenotypic parameters at the germination stage, but also in the specific physiological and biochemical processes (Li et al., 2010; Jian et al., 2016; Channaoui et al., 2019). In the next research, it will be necessary to further explore and improve the comprehensive evaluation method of canola drought tolerance with combining physiological characters which could be measured easily and conveniently. 


\section{CONCLUSIONS}

Based on the principal component analysis of the drought tolerance coefficients of 16 characters of the 53 canola varieties, the original single characters related to drought tolerance were transformed into six independent comprehensive indexes, representing $87.5 \%$ of the total related data in this experiment. The comprehensive evaluation value (D-value) of each variety was calculated by means of the membership function and cluster analysis. The 53 varieties could be divided into three categories, five varieties with higher drought tolerance relatively, 16 varieties with medium drought tolerance and the rest 32 ones with relatively lower drought tolerance.

By stepwise regression analysis, six drought resistance indexes were selected, such as dry weight per plant, fresh weight per plant, germination index, mean seedling emergence time, 1000-seeds weight and shoot dry weight per plant, and the optimal regression equation was established, which made the prediction quicker and more convenient for canola varieties difference in drought tolerance. Therefore, the integrated method including the principal component analysis, cluster analysis and stepwise regression analysis was more reliable for the comprehensive evaluation of drought tolerance difference during canola germination and emergence period. It not only avoided the one-sidedness and instability of the single indicator evaluation, but also revealed the relationship between drought tolerance coefficients and characters related drought tolerance in canola germination and emergence stage.

\section{ACKNOWLEDGEMENTS}

The research and publication were supported by the National Natural Science Foundation of China (31660354), Program for Top Talents in Science and Technology of Education Department in Guizhou (KY [2016]108) and Scientific Research Foundation for Doctors of Tongren University (trxyDH1608).

\section{REFERENCES}

Aksenova, L.A., Dunaeva, M.V., Zak, E.A., Osipov, Y.F., and Klyachko, N.L. 1994. The effect of tween-80 on seed-germination in winter-wheat cultivars differing in drought resistance. Russian Journal of Plant Physiology 41:557-559.

Ali, Q., Javed, M.T., Noman, A., Haider, M.Z., Waseem, M., Iqbal, N., et al. 2018. Assessment of drought tolerance in mung bean cultivars/lines as depicted by the activities of germination enzymes, seedling's antioxidative potential and nutrient acquisition. Archives of Agronomy and Soil Science 64:84-102.

Bai, P., Ran, C., and Xie, X. 2014. Influence of drought stress on physiological characteristics and agronomic traits at bud stage of rapeseed (Brassica napus L.) Scientia Agricultura Sinica 47:3566-3576.

Borisjuk, L., Neuberger, T., Schwender, J., Heinzel, N., Sunderhaus, S., Fuchs, J., et al. 2013. Seed architecture shapes embryo metabolism in oilseed rape. Plant Cell 25:1625-1640.

Byzova, M., Verduyn, C., Brouwer, D.D., and Block, M.D. 2004. Transforming petals into sepaloid organs in Arabidopsis and oilseed rape: implementation of the hairpin RNA-mediated gene silencing technology in an organ-specific manner. Planta 218:379-387.

Channaoui, S., Idrissi, I.S., Mazouz, H., and Nabloussi, A. 2019. Reaction of some rapeseed (Brassica napus L.) genotypes to different drought stress levels during germination and seedling growth stages. OCL - Oilseeds and Fats, Crops and Lipids 26:23.

Farid, M., and Ridwan, I. 2018. Tolerance limits of Indonesian rice varieties to drought and salinity in germination phase using PEG and $\mathrm{NaCl}$ as selection agents. IOP Conference Series: Earth and Environmental Science 157:012011.

Hamzei, J., and Soltani, J. 2012. Deficit irrigation of rapeseed (Brassica napus L.) for water-saving: Effects on biomass accumulation, light interception and radiation use efficiency under different $\mathrm{N}$ rates. Agriculture Ecosystems \& Environment 155:153-160.

Jian, H.J., Wang, J., Wang, T.Y., Wei, L.J., Li, J., and Liu, L.Z. 2016. Identification of rapeseed (Brassica napus L.) microRNAs involved in early stage seed germination under salt and drought stresses. Frontiers in Plant Science 13:658.

Khodarahmpour, Z., and Motamedi, M. 2011. Evaluation of drought and salinity stress effects on germination and early growth of two cultivars of maize (Zea mays L.) African Journal of Biotechnology 10:14868-14872.

Kuai, J., Sun, Y.Y., Zhou, M., Zhang, P.P., Zuo, Q.S., Wu, J.S., et al. 2016. The effect of nitrogen application and planting density on the radiation use efficiency and the stem lignin metabolism in rapeseed (Brassica napus L.) Field Crops Research 199:89-98.

Li, L., Li, J.G., Shen, M.C., Zhang, C.L., and Dong, Y.H. 2015. Cold plasma treatment enhances oilseed rape seed germination under drought stress. Scientific Reports 5:13033.

Li, Z., Lu, G.Y., Zhang, X.K., Zou, C.S., Cheng, Y., and Zheng, P.Y. 2010. Improving drought tolerance of germinating seeds by exogenous application of gibberellic acid $\left(\mathrm{GA}_{3}\right)$ in rapeseed (Brassica napus L.) Seed Science and Technology 38:432-440. 
Liu, J., Hasanuzzaman, M., Wen, H.L., Zhang, J., Peng, T., Sun, H.W., et al. 2019a. High temperature and drought stress cause abscisic acid and reactive oxygen species accumulation and suppress seed germination growth in rice. Protoplasma 256:1217-1227.

Liu, Y., Xu, H., Wen, X.X., and Liao, Y.C. 2016. Effect of polyamine on seed germination of wheat under drought stress is related to changes in hormones and carbohydrates. Journal of Integrative Agriculture 15:2759-2774.

Liu, S.T.,Zenda, T., Dong, A.Y., Yang, Y.T.,Liu, X.Y., Wang, Y.F., et al. 2019b. Comparative proteomic and morpho-physiological analyses of maize wild-type Vp16 and mutant vp16 germinating seed responses to PEG-induced drought stress. International Journal of Molecular Sciences 20(22):5586.

Maataoui, A., Talouizte, A., Benbella, M., and Bouhache, M. 2005. Effect of plant density on competitiveness of Brassica napus, Sinapis alba and S. arvensis under water stress conditions. Communications in Agricultural and Applied Biological Sciences 70:61-66.

Marcinkeviciene, A., Velicka, R., Raudonius, S., and Kosteckas, R. 2013. The relationship between root biomass and productivity of spring oilseed rape (Brassica napus L.) as influenced by crop density and fertilization. Zemdirbyste-Agriculture 100:39-44 .

Michel, B.E., and Kaulmann, M. 1973. The osmotic potential of polyethylene glycol 6000. Plant Physiology 51:914-91.

Muller, J., Behrens, T., and Diepenbrock, W. 2005. Measurement and modelling of canopy gas exchange of oilseed rape. Agricultural and Forest Meteorology 132:181-200.

Nourgholipour, F., Hosseini, H.M., Tehrani, M.M., Motesharezadeh, B., and Moshiri, F. 2018. Comparison of phosphorus efficiency among spring oilseed rape cultivars in response to phosphorus deficiency. New Zealand Journal of Crop and Horticultural Science 46(1):54-71.

Pavia, I., Rocha, L., Moutinho-Pereira, J., Lima-Brito, J., and Correia, C. 2019. Screening for drought resistance during germination of modern and old Iberian wheat cultivars. Acta Botanica Croatica 78:169-174.

Shabani, A., Sepaskhah, A.R., and Kamgar-Haghighi, A.A. 2014. Estimation of yield and dry matter of rapeseed (Brassica napus L.) using logistic model under water salinity and deficit irrigation. Archives of Agronomy and Soil Science 60:951-969.

Toscano, S., Romano, D., Tribulato, A., and Patane, C. 2017. Effects of drought stress on seed germination of ornamental sunflowers. Acta Physiologiae Plantarum 39:184.

Xie, X.Y., Zhang, X., and He, Q.L. 2013. Identification of drought resistance of rapeseed (Brassica napus L.) during germination stage under PEG stress. Journal of Food Agriculture \& Environment 11:751-756.

Yin, Y., and Wang, H. 2012. Achievement, problem and scientific policy of rapeseed (Brassica napus L.) industry development in China. Review of China Agricultural Science and Technology 14:1-7.

Zhang, H.P., Berger, J.D., and Milroy, S.P. 2013a. Genotype $\times$ environment interaction studies highlight the role of phenology in specific adaptation of canola (Brassica napus L.) to contrasting Mediterranean climates. Field Crops Research 144:77-88.

Zhang, J., Cui, Y., Zhang, L.Y., Wang, Y.L., Li, J., Yan, G.J., et al. 2013b. Seed coat color determines seed germination, seedling growth and seed composition of canola (Brassica napus L.) International Journal of Agriculture and Biology 15:535-540.

Zhao, J.Y., Becker, H.C., Zhang, D.Q., Zhang, Y.F., and Ecke, W. 2005. Oil content in a European $\times$ Chinese rapeseed (Brassica napus L.) population: QTL with additive and epistatic effects and their genotype-environment interactions. Crop Science 45:51-59. 